\title{
Pairing and specific heat in hot nuclei
}

\author{
Danilo Gambacurta* \\ GANIL, CEA and CNRS/IN2P3, Boîte Postale 55027, 14076 Caen Cedex, France
}

Denis Lacroix

Institut de Physique Nucléaire, IN2P3-CNRS, Université Paris-Sud, F-91406 Orsay Cedex, France and GANIL, CEA/DSM and CNRS/IN2P3, Boîte Postale 55027, $140 \% 6$ Caen Cedex, France

\author{
N. Sandulescu甪 \\ National Institute of Physics and Nuclear Engineering, P.O.Box MG-6, Magurele-Bucharest, Romania
}

\begin{abstract}
The thermodynamics of pairing phase-transition in nuclei is studied in the canonical ensemble and treating the pairing correlations in a finite-temperature variation after projection BCS approach (FT-VAP). Due to the restoration of particle number conservation, the pairing gap and the specific heat calculated in the FT-VAP approach vary smoothly with the temperature, indicating a gradual transition from the superfluid to the normal phase, as expected in finite systems. We have checked that the predictions of the FT-VAP approach are very accurate when compared to the results obtained by an exact diagonalization of the pairing Hamiltonian. The influence of pairing correlations on specific heat is analysed for the isotopes ${ }^{161,162} \mathrm{Dy}$ and ${ }^{171,172} \mathrm{Yb}$. It is shown that the FT-VAP approach, applied with a level density provided by mean field calculations and supplemented, at high energies, by the level density of the back-shifted Fermi gas model, can approximate reasonably well the main properties of specific heat extracted from experimental data. However, the detailed shape of the calculated specific heat is rather sensitive to the assumption made for the mean field.
\end{abstract}

PACS numbers: 25.60.Je,25.40.Hs,24.30.Cz,21.10.Re,21.60.Jz

Keywords: pairing, thermodynamics, nuclear models

\section{INTRODUCTION}

In the last decade there is a renewed interest for studying the fingerprints of pairing correlations in the thermodynamic properties of excited nuclei. This interest was triggered by the new accurate measurements of level density at low excitation energies. Thus, a special attention was payed lately to the influence of pairing on the low-temperature behavior of the specific heat in the isotopes ${ }^{161,162} \mathrm{Dy}$ and ${ }^{171,172} \mathrm{Yb}$, extracted from the level density measurements performed by the Oslo group [1]. The possible thermal signatures of pairing correlations in these rare earth isotopes have been studied either with schematic models or employing various approximations which go beyond the standard BCS approach. As it is by now well documented, due to its drawbacks, i.e., particle number fluctuation and quasiparticle parity mixing, the BCS theory is not well-suited to describe pairing effects in hot nuclei. One alternative to cure these drawbacks is to use the particle-number projected BCS approximation extended to finite temperature. How this approximation can be implemented for performing variation after projection calculations at finite temperature (FT-VAP) was recently discussed in Ref [2]. This approach will be applied here to investigate the effect of pairing on thermal

\footnotetext{
*Electronic address: gambacurta@ganil.fr

${ }^{\dagger}$ Electronic address: lacroix@ganil.fr

‡Electronic address: sandulescu@theory.nipne.ro
}

properties of Dy and $\mathrm{Yb}$ isotopes mentioned above.

In Ref. [3] it was argued that the S-shaped form of the specific heat in Dy and $\mathrm{Yb}$ isotopes is generated by the transition from the superfluid to the normal phase. This conclusion was drawn by the comparison with the specific heat of a non-interacting Fermi gas which was described by the back-shifted Bethe formula [4]. However, this comparison is misleading because the Bethe formula is not valid for low energy excitations (e.g., see [5]), where the pairing correlations are expected to be important. In this study we shall re-analyze this issue in the framework of FT-VAP approximation and using for low energy excitations level densities extracted from self-consistent mean field calculations.

The article has the following structure. First, we present the calculation scheme we use to evaluate the thermodynamic properties of nuclei related to the pairing interaction. Afterwards, using single-particle spectra generated by self-consistent mean field models, we analyze the effect of pairing on the partition function and heat capacity. Finally, a critical discussion on the comparison with experimental data is made.

\section{STATISTICAL TREATMENT OF PAIRING INTERACTION IN FINITE SYSTEMS}

To analyze the effects of pairing correlations upon the thermodynamic properties of hot nuclei we consider the 
hamiltonian:

$$
\hat{H}=\sum_{i} \epsilon_{i} a_{i}^{+} a_{i}+\sum_{i, j} V_{i j} a_{i}^{+} a_{\bar{i}}^{+} a_{\bar{j}} a_{j},
$$

where the second term is the pairing interaction which scatters pairs among time-reversed single-particle states $(i, \bar{i})$. This hamiltonian can be used for realistic description of pairing correlations in heavy nuclei for which proton-neutron pairing can be neglected.

For many years the pairing correlations in hot nuclei have been described in the framework of finitetemperature BCS/HFB models (FT-BCS/HFB) [7]. However, FT-BCS is the proper theory for infinite systems but not for finite systems such as atomic nuclei. This is reflected in the fact that FT-BCS predicts a sharp (second order) superfluid-normal phase transition, in contrast to a smooth transition, as expected in hot nuclei [2]. This drawback of FT-BCS is related to the improper treatment of particle number conservation, pleading in favor of approaches that explicitly conserve particle number. Several calculation schemes have been proposed along this line, e.g., based on Shell-Model Monte-Carlo (SMMC) calculations [6] or the method proposed recently in Ref. [9]. In the present study we shall explore a variational method which is the generalization of the particlenumber projected BCS model to finite temperature. This method, called below finite-temperature variation after projection (FT-VAP) approach, has been recently tested [2, 11] in the case of schematic pairing models. Here the FT-VAP approach will be extended and applied to describe thermodynamic properties of hot nuclei. In order to test the validity of FT-VAP in realistic applications, we perform also thermodynamic calculations based on the spectrum of the pairing Hamiltonian (1) obtained by direct diagonalization.

\section{A. Input for pairing Hamiltonian}

In the applications presented below the single-particle (s.p.) states employed in the Hamiltonian (11) are taken from mean-field models. Thus, we have used two sets of s.p. states obtained, respectively, from Skyrme-HartreeFock (HF) and Relativistic Mean-Field (RMF) calculations allowing, in both cases, axial symmetry deformation. The Skyrme-HF equations are solved with the code EV8 [12] and using the force Sly4 [13] while the RMF calculations are done with the force PK1 [14]. The s.p. energies around the Fermi energy for the isotopes ${ }^{162}$ Dy and ${ }^{172} \mathrm{Yb}$ obtained in Skyrme-HF and RMF [15] calculations are shown in Figure 1. These energies are also considered in the calculations done for the odd isotopes ${ }^{161} \mathrm{Dy}$ and ${ }^{171} \mathrm{Yb}$. In HF and RMF calculations a deformation of about $\beta_{2}=0.35$ is found both for ${ }^{162} \mathrm{Dy}$ and ${ }^{172} \mathrm{Yb}, \beta_{2}$ being defined by

$$
\beta_{2}=\left(\frac{5 \pi}{9}\right)^{\frac{1}{2}} \frac{\left\langle\hat{Q}_{2}\right\rangle}{A R_{0}^{2}}
$$

where $A$ denotes the mass number, $R_{0}=1.2 A^{1 / 3}$ and $\hat{Q}_{2}$ is the quadrupole operator.

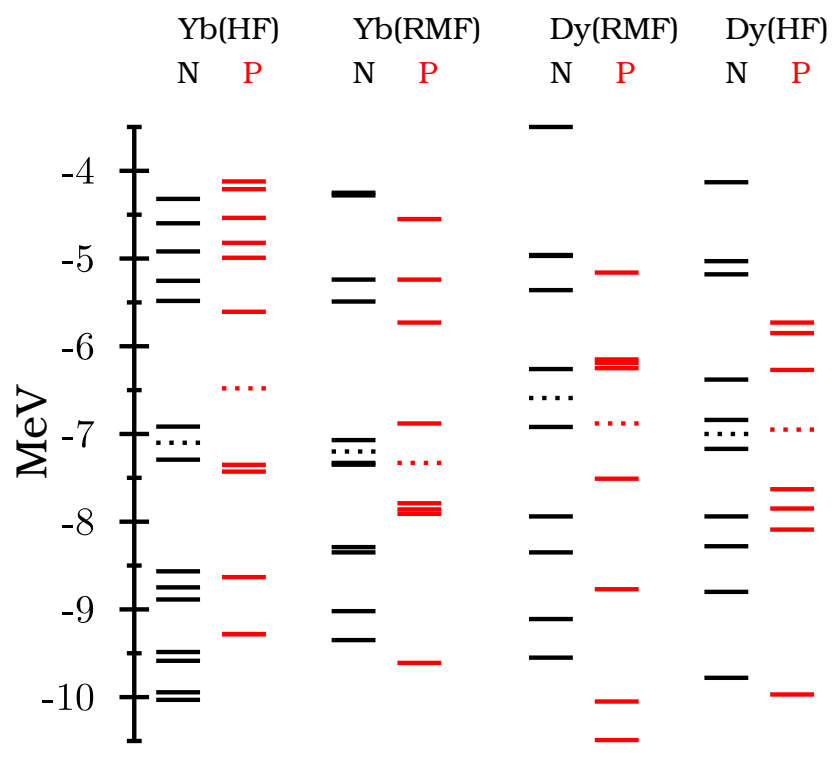

FIG. 1: (color online) Neutrons (N) and protons (P) single particle energies around the Fermi energies (dotted lines) for ${ }^{162} \mathrm{Dy}$ and ${ }^{172} \mathrm{Yb}$ obtained from HF-Sly4 and RMF-PK1 calculations [15].

As commonly done in BCS calculations at zero temperature, pairing interaction is considered acting in a limited energy window around the Fermi level. In this window the matrix elements of the pairing interaction are taken equal to a constant strength, i.e., $V_{i j}=G$. In the calculations presented below for Dy and $\mathrm{Yb}$ isotopes we have considered an energy window of $3 \mathrm{MeV}$. The pairing strength $G$ is fixed at BCS level in order to give a pairing gap approximately equal to $0.8 \mathrm{MeV}$ both for protons and neutrons. The number of single particle states and the active nucleons as well as the corresponding pairing strength $G$ for the energy window of $3 \mathrm{MeV}$ are given in Table 【

\section{B. Pairing treatment in canonical ensemble}

The thermodynamic properties of pairing interaction are calculated here in the canonical ensemble [8]. The key quantity is the canonical partition function

$$
Z(\beta)=\operatorname{Tr} e^{-\beta \hat{H}} \equiv \operatorname{Tr}\left(D_{N}[\beta]\right),
$$

where $\beta=1 / T$ and $D_{N}[\beta]$ denotes the statistical $N$ body density operator. The trace has to be taken over the states with well-defined proton and neutron numbers. Using the eigenstates $\left\{E_{n}\right\}$ of the hamiltonian $H$, the partition function can be written as 23. 


\begin{tabular}{|c|c|c|c|c|}
\hline & \multicolumn{4}{|c|}{ Neutron } \\
\hline & $\mathrm{Yb}(\mathrm{HF})$ & $\mathrm{Yb}(\mathrm{RMF})$ & Dy (RMF) & $\mathrm{Dy}(\mathrm{HF})$ \\
\hline$N_{s p}$ & 14 & 11 & 10 & 10 \\
\hline$n_{a}$ & 16 & 12 & 10 & 10 \\
\hline$G(M e V)$ & 0.260 & 0.270 & 0.320 & 0.284 \\
\hline \multicolumn{5}{|c|}{ Proton } \\
\hline & $\mathrm{Yb}(\mathrm{HF})$ & $\mathrm{Yb}(\mathrm{RMF})$ & Dy (RMF) & $\mathrm{Dy}(\mathrm{HF})$ \\
\hline$N_{s p}$ & 11 & 8 & 8 & 9 \\
\hline$n_{a}$ & 10 & 8 & 8 & 8 \\
\hline$G(M e V)$ & 0.332 & 0.345 & 0.385 & 0.327 \\
\hline
\end{tabular}

TABLE I: Number of single particle levels $\left(N_{s p}\right)$ and active nucleons $\left(n_{a}\right)$ in the active $3 \mathrm{MeV}$ window around the Fermi energy for neutrons (upper table) and protons (lower table). The strength $G$ is fixed in order to have a pairing BCS gap of $0.8 \mathrm{MeV}$ for both the neutrons and protons.

$$
Z=\sum_{n} \rho\left(E_{n}\right) e^{-\beta E_{n}}
$$

where $\rho\left(E_{n}\right)$ stands for the level density. Quantities of physical interest are obtained from the derivatives of the partition function. For instance, the thermal energy can be evaluated through

$$
\begin{aligned}
E(T) & =-\frac{\partial \ln Z}{\partial \beta}=\langle H\rangle \\
& =Z^{-1} \sum_{n} \rho\left(E_{n}\right) E_{n} e^{-\beta E_{n}},
\end{aligned}
$$

where $\langle X\rangle=\operatorname{Tr}\left(X D_{N}[\beta]\right)$.

In the case of the Hamiltonian (1), using a pairing force restricted to a narrow window around the Fermi level, one can obtain the eigen-energies $E_{n}$ by direct diagonalization in subspaces of given seniority. Such approach is certainly the most direct way to describe statistical properties of pairing at fixed particle number. However, this approach can be only applied at low temperatures because only in this case the pairing active window can be taken small enough for allowing exact diagonalization. Alternatively, the statistical properties of pairing interaction are usually investigated in the framework of quasiparticle models, such as BCS or HFB, in which the excitations can be easily obtained and the pairing gap is built from outset. Thus, in the finite-temperature BCS (FTBCS) approximation the partition function (3) is calculated with the BCS effective Hamiltonian while the variation of the pairing correlations with the temperature are characterized by the pairing gap (see for example [21]). For illustration, in the top panel of Fig. 2 it is shown how the pairing gap varies with the temperature in the isotopes ${ }^{162} \mathrm{Dy}$ and ${ }^{172} \mathrm{Yb}$. The results corresponds to the RMF s.p. spectrum. As can be seen, the FT-BCS predicts a sharp second order type transition from the superfluid to the normal phase, at variance with what it

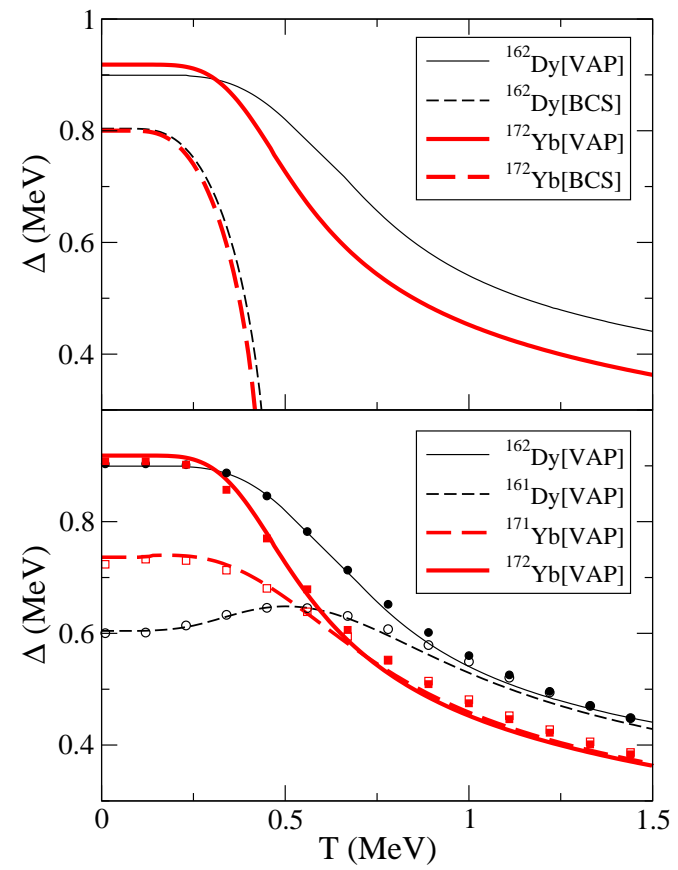

FIG. 2: (color online) Top: neutron pairing gap as a function of temperature for ${ }^{162} \mathrm{Dy}$ and ${ }^{172} \mathrm{Yb}$ obtained in FT-VAP and FT-BCS approximations. Bottom: comparison between the pairing gaps in odd-even and even-even $\mathrm{Dy}$ and $\mathrm{Yb}$ isotopes obtained in FT-VAP approximation. By symbols are shown the gaps corresponding to exact diagonalization, calculated with formula (7).

is expected for small finite systems. As it is well known, the sharp transition predicted by FT-BCS is connected to the breaking of particle number conservation, leading automatically to a grand-canonical treatment instead of a canonical one.

A more involved but more appropriate approach, which keeps the physical insight of quasiparticle models, can be obtained working with the particle number projected density

$$
\hat{D}_{N}=\frac{1}{Z} \hat{P}_{N} \exp (-\beta \hat{h}) \hat{P}_{N}
$$

where $Z=\operatorname{Tr}\left(\hat{P}_{N} \exp (-\beta \hat{h}) \hat{P}_{N}\right), \hat{h}$ is the BCS effective Hamiltonian, and $\hat{P}_{N}$ is the projector onto good particle number. This density is used below in a variation after projection (VAP) scheme to minimize the Helmholtz free energy. This approach has been proposed already some times ago [11] and recently tested in the Richardson model [2]. Technical details related to the solution of the FT-VAP equations can be found in the latter reference.

An illustration of the pairing gap obtained using the 
FT-VAP approach can be seen in the top panel of Fig. 2 where are shown the results for the isotopes ${ }^{162} \mathrm{Dy}$ and ${ }^{172} \mathrm{Yb}$. Contrary to the FT-BCS approximation, FTVAP predicts a smooth transition from the superfluid to to the normal phase, with a non-vanishing pairing gap extended up to high temperatures. To test the predictions of FT-VAP we have also done calculations using the exact solutions of the pairing Hamiltonian obtained by direct diagonalization and, similarly to ref. [2], we have estimated the pairing gap by the formula

$$
\Delta=\sqrt{-G\left(E-E_{0}\right)}
$$

where $E$ is the total exact energy and $E_{0}$ is given by

$$
E_{0}=\sum_{i}\left(\varepsilon_{i}-\frac{G}{2} n_{i}\right) n_{i}
$$

The s.p. occupation numbers $n_{i}$ are those deduced from the exact calculation. The gap obtained by the exact diagonalization and the FT-VAP are compared in the bottom part of Fig. 2. As can be seen, the two calculations give similar gaps, indicating the high accuracy of the FT-VAP approach. In Fig. 2 are given also the pairing gaps obtained for the odd nuclei. As expected, at low $T$, the gaps in odd systems are smaller than in the even systems. This difference tends to disappear as the temperature increases. For ${ }^{161} \mathrm{Dy}$ and to a lesser extend for ${ }^{171} \mathrm{Yb}$, we also observe a small re-entrance effect around $T=0.5 \mathrm{MeV}$ (resp. around 0.2-0.3 MeV). This effect is related to the thermal scattering of the odd particle in high energy s.p. levels, which increases the available phase space for the pair scattering.

\section{HEAT CAPACITY}

Statistical properties of hot nuclei are commonly described by the heat capacity extracted from experimental level density. The specific heat is calculated from the second derivative of the partition function

$$
C_{V}(T)=\beta^{2} \frac{\partial^{2} \ln Z}{\partial^{2} \beta} .
$$

The partition function and the specific heat for ${ }^{162} \mathrm{Dy}$, obtained with the s.p. spectrum truncated by a $3 \mathrm{MeV}$ energy window around the Fermi level, are shown in Fig. 3 by the lines labeled "int,tr". For comparison are also shown, by the lines labeled "nint,tr", the results corresponding to the free spectrum $(\mathrm{G}=0)$. It can be seen that, in both calculations, the specific heat has an unphysical behaviour at high temperatures. To cure this behaviour one should considering s.p. states from energy windows around the Fermi energy larger than $3 \mathrm{MeV}$. Since the pairing force is of zero range, in principle the effect of pairing correlations can be study with any energy window provided the strength of the force is adjusted such as to preserve the amount of pairing correlations, measured, for instance, by the BCS pairing gap. However, this strategy cannot be applied for too large energy windows because the number of excited states which can be built on from the s.p. levels becomes too large to be handled in the FT-VAP or direct diagonalization calculations. The calculations can be, however, simplified by taking into account the fact that the pairing correlations have little effect on the excitations of high energies. Based on this argument, we adopt here the calculation scheme proposed in Ref. [6] and assume that the pairing interaction affects the excitation energies generated by the s.p. energies taken from a restricted region around the Fermi energy while the rest of s.p. spectrum is treated as in the case of non-interacting particles. For the partition function this approximation can be written as $[6]$ :

$$
\ln Z_{i n t}^{\prime}=\ln Z_{i n t, t r}^{\prime}-\ln Z_{n i n t, t r}^{\prime}+\ln Z_{n i n t}^{\prime},
$$

where $Z_{\text {int,tr }}^{\prime}$ and $Z_{\text {nint,tr }}^{\prime}$ are, respectively, the partition function calculated with and without pairing interactions in the truncated space, here the $3 \mathrm{MeV}$ window. $Z_{\text {nint }}^{\prime}$ is the partition function calculated without the pairing interaction considering all the s.p. levels from a window of $7 \mathrm{MeV}$ around the Fermi energy. We have checked that enlarging further this space does not affect the results in the temperature region considered below.

As an illustration of the use of formula (10), in top panel of figure 3 are shown the various contributions to the partition function for the case of ${ }^{162} \mathrm{Dy}$. The results obtained with the extended partition function are labeled by "int". For temperatures below $T \simeq 0.5 \mathrm{MeV}$ the canonical partition function for the non-interacting case $(G=0)$ is evaluated by a direct counting of the excited states. For higher temperatures, when this procedure becomes numerically difficult, the canonical partition function $Z_{n i n t}^{\prime}$ is evaluated, through the saddle point approximation, from the partition function in the grandcanonical ensemble. This approximation is discussed in Ref. [6] and the main formulas are given in appendix A It should be mentioned that this approximation does not work well at low temperatures, which is the reason why we apply it here for temperatures above $T \simeq 0.5 \mathrm{MeV}$.

From the partition function calculated as explained above we have obtained the heat capacity by applying Eq. (9). The results for ${ }^{162} \mathrm{Dy}$ are shown in the bottom panel of Fig. 3. This figure clearly shows that the calculations done in the truncated space become unreliable at higher temperatures. Indeed, from Fig. 3 one can notice that for temperatures larger than $T \simeq 0.5 \mathrm{MeV}$ the heat capacity calculated in the truncated space becomes much smaller than the specific heat evaluated with the extended partition function (10), which reflects the important contribution of high energy excitations which are artificially cut in the former case. On the other hand, as expected from the assumption we have made relative to the calculation of the extended partition function, it can be seen that the specific heats obtained with and without the pairing interaction become similar at high temperatures. 
${ }^{162}$ Dy

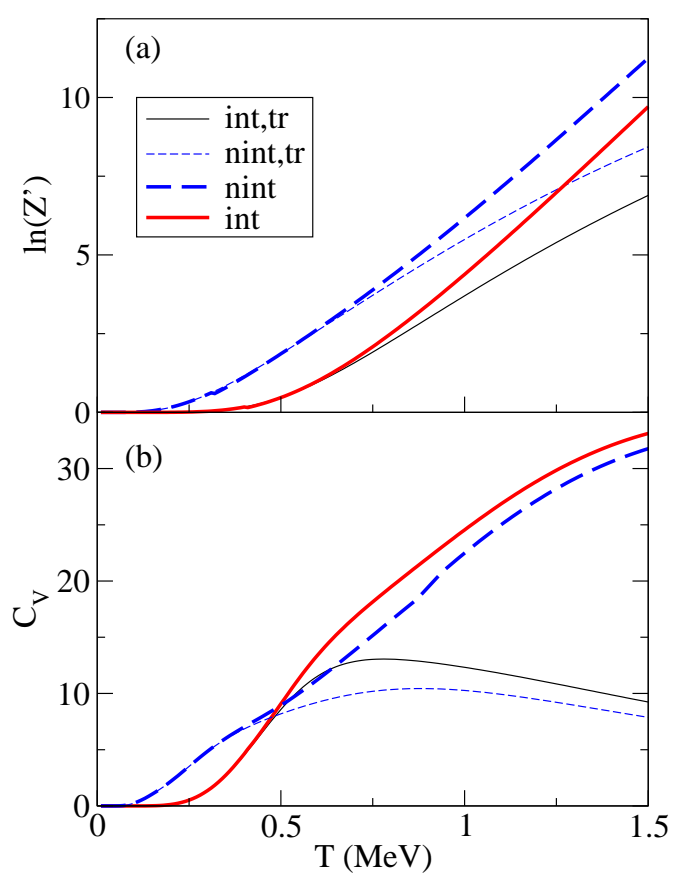

FIG. 3: (color online) Top: Partition functions for ${ }^{162}$ Dy. The labels "nint-tr" and "int-tr" correspond to the partition function obtained, respectively, without and with the pairing interaction and considering the single-particles states from a window of $3 \mathrm{MeV}$. The label "nint" corresponds to the case of non-interacting particles in the window of $7 \mathrm{MeV}$ while the results for the extended partition function $\ln Z^{\prime}$ (10) are labeled by "int". Bottom: Corresponding heat capacities.

The most important information which can be extracted from Fig. 3is the effect of pairing correlations on the specific heat. Comparing the results obtained with and without the pairing force, one can thus see that below $T \simeq 0.5 \mathrm{MeV}$ the specific heat is strongly suppressed by pairing correlations, an effect which can be traced back to the large pairing gap in the low temperature region (see Fig. 21). Above $T \simeq 0.5 \mathrm{MeV}$, the specific heat becomes larger than the results for non-interacting particles and then, at much higher temperatures, it goes closer to the latter. This dependence of the specific heat on temperature is commonly referred to as a "S-shape" behaviour.

We have also analyzed the effect of pairing on specific heat separately for protons and neutrons. The results for the isotopes ${ }^{161,162} \mathrm{Dy}$ and ${ }^{171,172} \mathrm{Yb}$ are given in Figs. 7 and 8 In these figures are shown the results obtained by applying, in the truncated space, the FT-VAP approximation and the method of direct diagonalization. It can be seen that both treatments give similar results, confirming again the good predictive power of the FTVAP approximation. From these figures we can observe that for even number of neutrons or protons there is a large difference between the non-interacting and interacting case, as noticed for the total specific heat of ${ }^{162} \mathrm{Dy}$ discussed above. However, for odd number of neutrons this difference is much smaller. Consequently, the effect of pairing on total specific heat in even-even and oddeven isotopes is different. This is illustrated in Figure 6. As expected, at large temperatures, for which the pairing correlations are not anymore effective, the difference between even-even and odd-even systems is becoming very small.

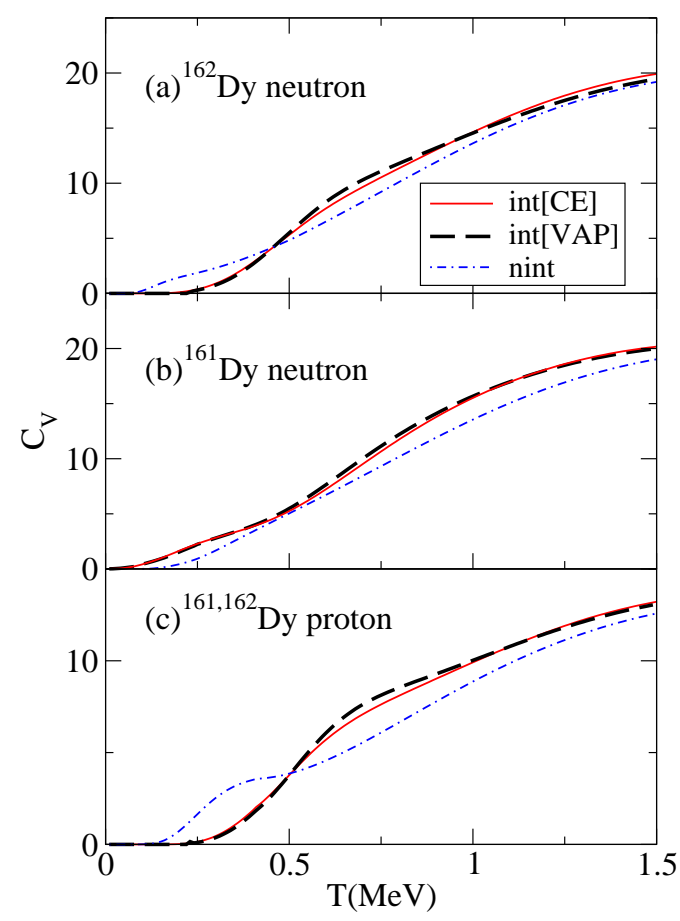

FIG. 4: (color online) Neutron specific heat for ${ }^{162}$ Dy (top), ${ }^{161} \mathrm{Dy}$ (middle) and the proton specific heat for ${ }^{162,161}$ Dy (bottom). The lines labeled by "int(VAP)" and "int(CE)" give the results corresponding to FT-VAP and, respectively, to exact diagonalization while "nint" are the results for noninteracting particles. The calculations are done with the extended partition function (10).

To check the convergence of the results with the energy window chosen for the pairing interaction, we have performed a second calculations by enlarging the interacting window from $3 \mathrm{MeV}$ to $5 \mathrm{MeV}$. In both calculations the strength of the pairing force was fixed in order to get a BCS gap equal to $\Delta=0.8 \mathrm{MeV}$ and the extended partition function was calculated with a window of $7 \mathrm{MeV}$. The results for the specific heat obtained for the two energy windows are shown in Fig. 7. It can be seen that the two calculations are on top of each other.

The specific heat calculated in the present approach depends on the assumptions made on single-particle energies and the strength of the pairing interaction employed 


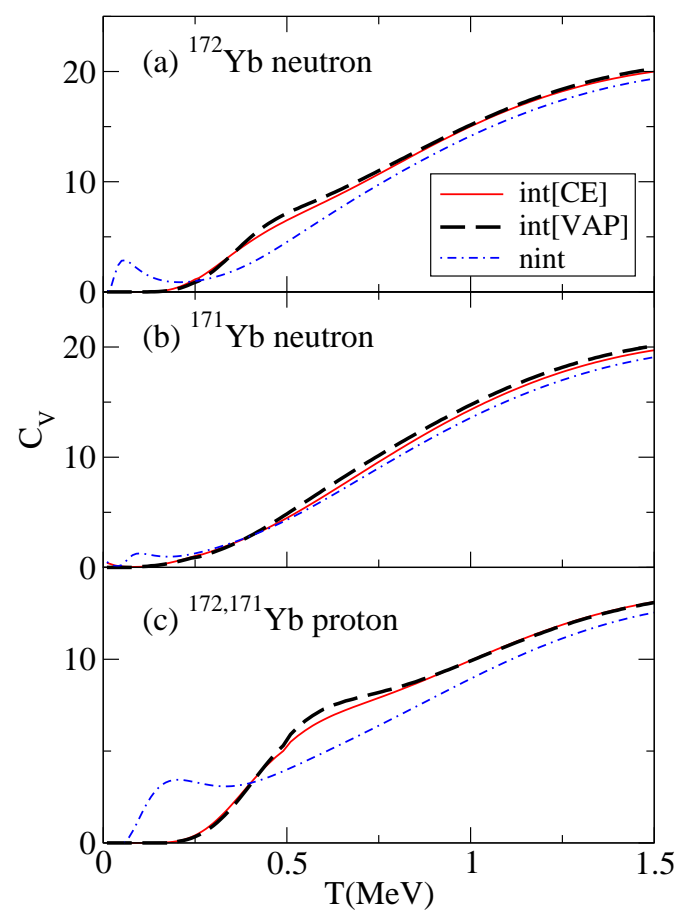

FIG. 5: (color online) The same as in Fig. 4 but for $\mathrm{Yb}$ isotopes.

in the Hamiltonian (1).

The sensitivity of the specific heat to the strength of the pairing interaction is illustrated in Fig. 8 where it is shown the specific heat of neutrons in ${ }^{162}$ Dy obtained with three values for the strength corresponding to the BCS gaps $\Delta=\{0.6,0.8,1.0\} \mathrm{MeV}$. As expected, the Sshape is becoming more pronounced when the strength of the force is increasing.

How much depends the specific heat on the singleparticle spectrum can be seen from Figs 9 and 10, where are compared the results obtained with the energies provided by the Skyrme-HF and the RMF calculations. In both cases the strength of the pairing force is adjusted to obtain the same BCS gap. It can be seen that there are significant differences between the results obtained with the two mean fields, especially for Yb isotopes. These differences are related to the distribution of the single particle levels around the Fermi energy, shown in Fig. 1. Consequently, a change of the pairing strength can be easily compensated by a change in the single particle energies, which makes the comparison with experimental data rather difficult, especially keeping in mind the current debate regarding the possibility for an energy density functional approach to be predictive for the effective single-particle energies [22].

As it has been observed in previous studies (e.g., see [18]), the presence of a bump or a S-shape in the heat capacity does not necessarily signs the transition from

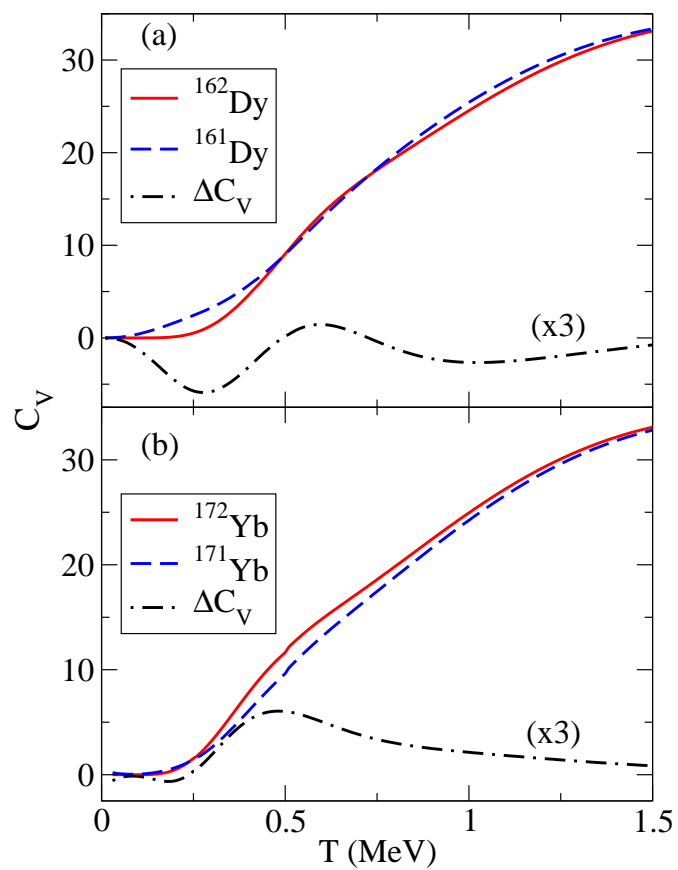

FIG. 6: (color online) Total (neutron+proton) heat capacity in even-even and odd-even Dy (top) and $\mathrm{Yb}$ (bottom) isotopes. The difference $\Delta C_{V}=C_{V}^{\text {even }}-C_{V}^{\text {odd }}$ is also shown by dot-dashed line (note that the difference is multiplied by a factor 3).

superfluid to normal system. This is clearly proved by the non-interacting case, where bumps are sometimes observed in the low temperature regime. Such a behavior is indeed expected if isolated single-particle states are lying very close to the Fermi energy and a gap in energy exist between these states and other surrounding states. This situation happens for instance in the case of $\mathrm{Yb} \mathrm{nu}-$ clei for neutrons (see Fig. 1 (left)) and is reflected by a pronounced bumps at low temperature (Figure 5).

To be more quantitative, let us consider the schematic situation of a set of $N$ degenerated two-level system centered around the Fermi energy. The system is assumed to be isolated from the other states. Then, the noninteracting hamiltonian can be written as:

$$
H=\sum_{k=1, N} \frac{\Delta \varepsilon}{2}\left(a_{2, k}^{\dagger} a_{2, k}-a_{1, k}^{\dagger} a_{1, k}\right)
$$

For this system, the canonical partition function writes:

$$
Z(\beta)=2^{N}\left[\cosh \left(\beta \frac{\Delta \varepsilon}{2}\right)\right]^{N},
$$

leading to:

$$
C_{V}=N\left(\beta \frac{\Delta \varepsilon}{2}\right)^{2}\left[1-\tanh ^{2}\left(\beta \frac{\Delta \varepsilon}{2}\right)\right] .
$$




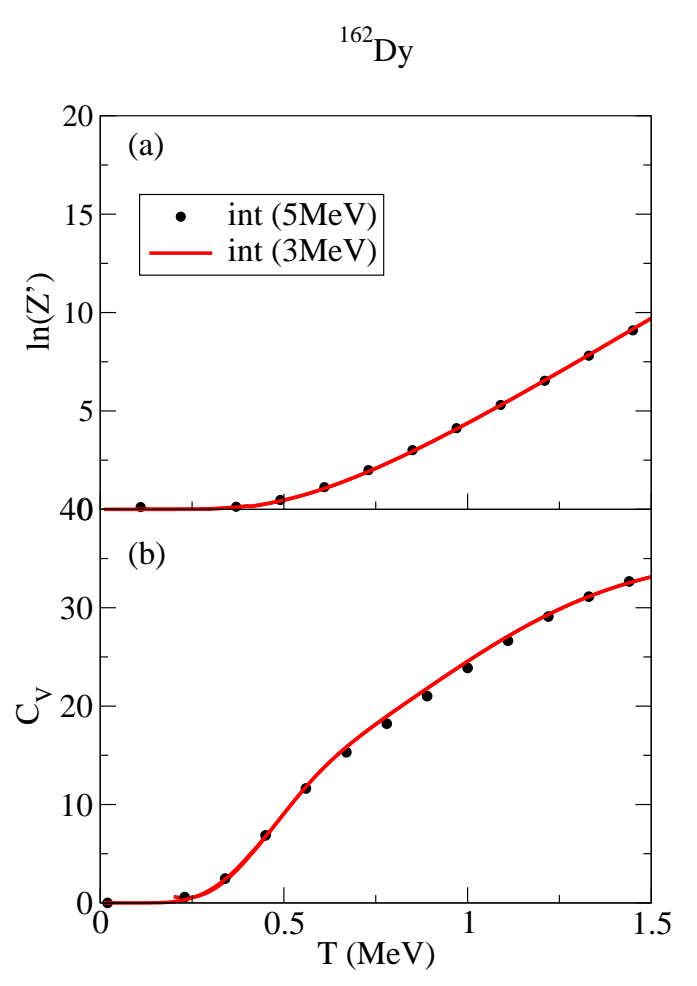

FIG. 7: (color online) Comparison between the partition functions and the heat capacities in ${ }^{162}$ Dy obtained with the pairing interaction acting in two energy windows, of $3 \mathrm{MeV}$ and $5 \mathrm{MeV}$. The calculations are done with the extended partition function (10) and fixing the pairing strength such as to get the same BCS pairing gap in the two energy windows.

Independently of the state degeneracy $N$, this heat capacity has a maximum at $\beta \Delta \varepsilon / 2 \simeq 1.2$, leading approximately to $T \simeq 0.417 \Delta \varepsilon$. This formula can give grossly indications on the possible appearance of the lowest peak in the low temperature region. For instance, from Fig. 1, we can deduce that $\Delta \varepsilon_{n} \simeq 0.26 \mathrm{MeV}$ (Yb nuclei with RMFPK1) leading to an expected bump around $0.1 \mathrm{MeV}$, that is consistent with the bump observed in upper panel of Fig. 5 which appears at $0.07-0.08 \mathrm{MeV}$.

\section{COMPARISON WITH EXPERIMENT}

The specific heats for Dy and $\mathrm{Yb}$ isotopes have been evaluated from the experimental level density in Refs [16, 17]. How these results compare with the present calculations, in the case of s.p. energies generated by RMF can be seen in Fig. 11. This figure shows that the calculated heat capacities underestimate significantly the experimental results. At first glance, one might conclude that the theory cannot quantitatively describe the experiments. However, it should be kept in mind that in Refs. [16, 17] the heat capacities are obtained from the level density using specific assumptions. More precisely, in

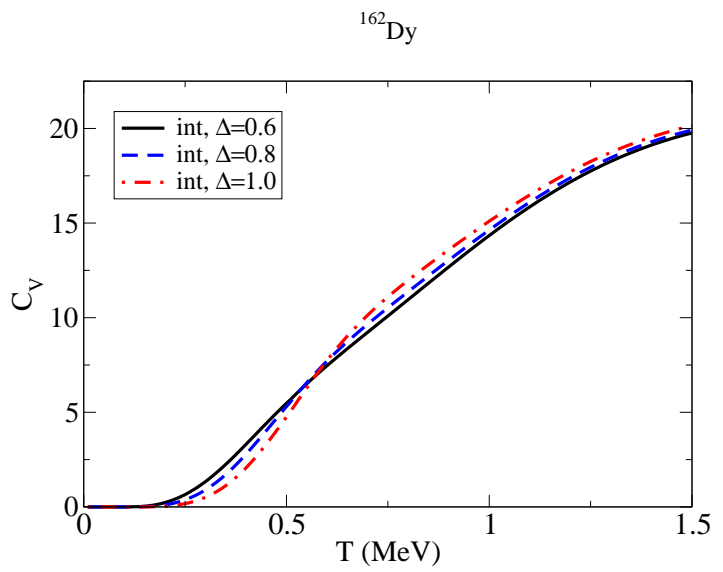

FIG. 8: (color online) Heat capacity in ${ }^{162}$ Dy for three different strengths of pairing interaction corresponding to the BCS gaps $\Delta=\{0.6,0.8,1.0\} \mathrm{MeV}$.

Refs. [16, 17] it is used the experimental level density for excitations below $8 \mathrm{MeV} /$ nucleon while for higher excitation energies it is employed the level density provided by the Back Shifted Fermi Gas Model (BSFGM) (Eq. (5) of [17]). In the present calculations, we have used for all excitations energies the level density generated by a discrete set of single-particle states. This assumption is expected to work reasonable well for low energy excitations but not for high energy excitations for which the contribution of the continuum become important. Thus the underestimation of the $C_{V}$ at high temperatures by the theoretical calculations appears to be related to the underestimation of the level density compared to the BSFGM.

To check that the difference between theory and experiment originates mainly from the different treatment of level densities at high excitation energies, where the pairing interaction is expected to not contribute, we have calculated the specific heat with the partition function Eq. (10) obtained using $Z_{\text {nint }}$ evaluated with BSFGM. The results are shown in Fig. 11. For comparison, for even isotopes we show also the specific heats obtained in the FT-BCS approach, calculated with $Z_{\text {nint }}$ generated by BSFGM, which present the unphysical sharp transition between the superfluid and the normal phase. It can be observed that the agreement of the FT-VAP results with the experiments is greatly improved. In particular, the calculated specific heat is now joining the experimental results at high temperatures. Globally, the S-shape behaviour of the specific heat seems to be more pronounced than in the calculations. However, we should keep in mind that, as we have discussed above, the shape of the calculated specific heat is rather sensitive to the assumption made for the mean field and to the strength of the interaction. This can be further seen in Fig. 12, where the calculated specific heats obtained by using the RMF and HF single-particle levels are compared to the 


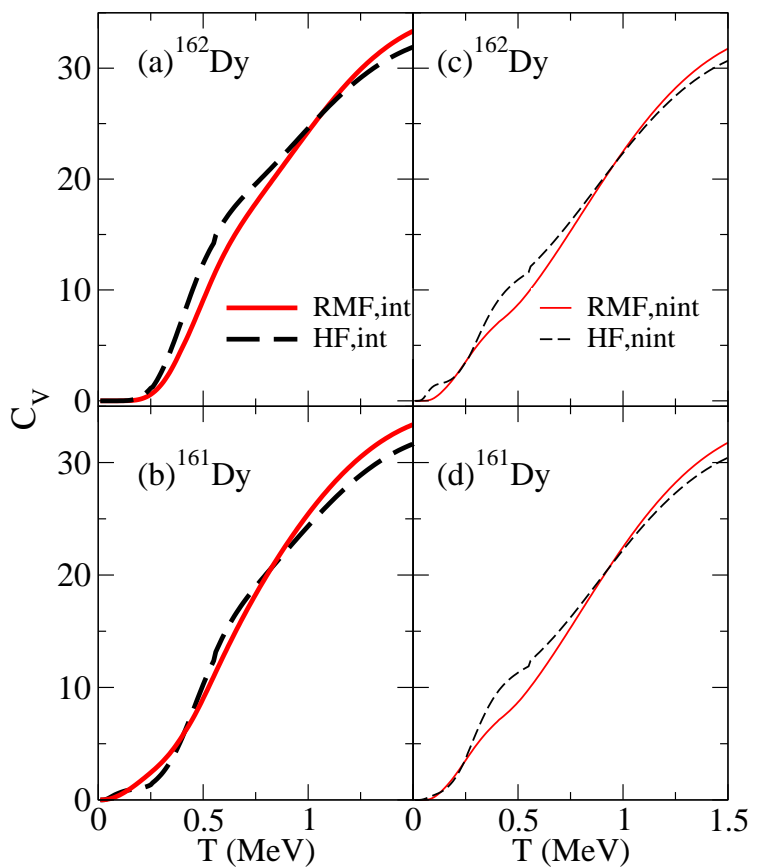

FIG. 9: (color online) Heat capacities in ${ }^{162}$ Dy and ${ }^{161}$ Dy obtained using two different sets of single-particle energies corresponding to RMF and HF mean fields. In the left (right) panels are shown the results with (without) the pairing interaction included. The results with the pairing interaction correspond to a BCS gap of $0.8 \mathrm{MeV}$.

experimental data. It can be noticed that the larger differences are found for the $\mathrm{Yb}$ isotopes. These differences in the calculated specific heat are generated mainly by the different proton level density around the Fermi level predicted by the HF and RMF calculations (see Fig. 1).

It should be mentioned that a conclusion on the validity of RMF compared to HF would be erroneous. Indeed, changing the Skyrme functional in HF and/or the effective interaction in RMF would completely change the level scheme and the comparison with experiments.

\section{CONCLUSION}

In the present paper we have studied the effect of pairing correlations on specific heat of hot nuclei in the framework of a variation after particle-number projection BCS formalism extended to finite temperature (FT-VAP). The calculation are done in canonical ensemble and with a Hamiltonian composed of a single-particle term, generated by Skyrme-HF or RMF calculations, and a pairing interaction of seniority type. The pairing interaction is considered active in a limited window around the Fermi level. The contribution of the states from the pairing

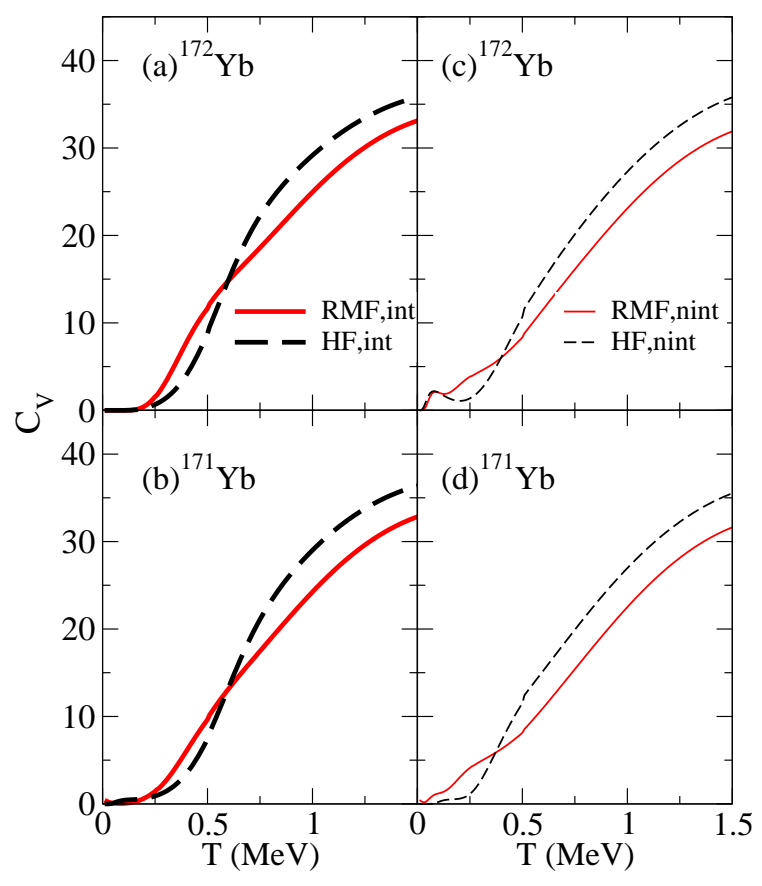

FIG. 10: (color online)The same as in Fig. 9 but for ${ }^{172} \mathrm{Yb}$ and ${ }^{171} \mathrm{Yb}$.

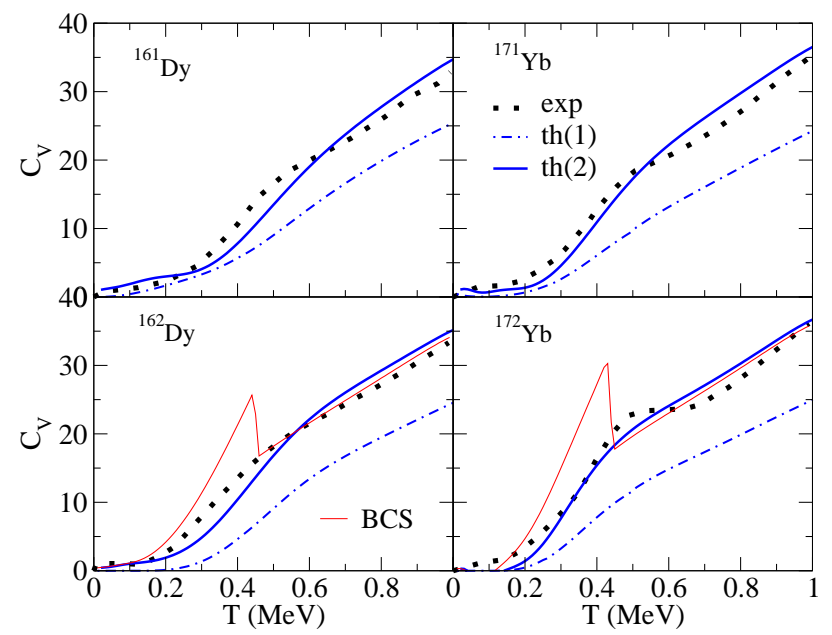

FIG. 11: (color online) Comparison between the calculated specific heats and the specific heats extracted from experimental data [16, 17]. By "th(1)" are indicated the results obtained with the level density generated by the single-particle spectrum of RMF. The results "th(2)" are obtained employing in Eq. (10) the partition function $\ln Z_{\text {nint }}^{\prime}$ generated by the back shifted Fermi gas model. For comparison, we show also the results obtained within the FT-BCS approximation . 


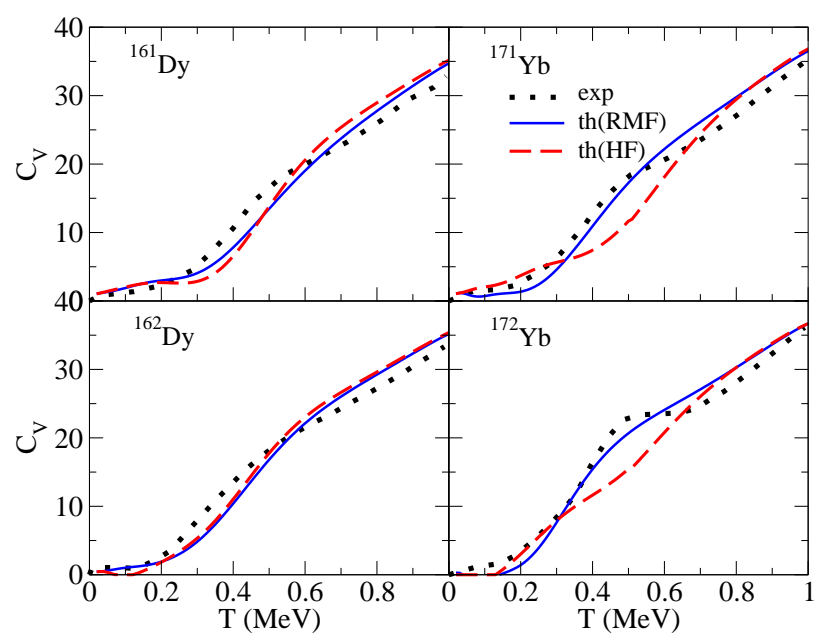

FIG. 12: (color online) Comparison between the specific heats obtained with the HF and RMF mean fields and with the extended partition function (10) calculated with $\ln Z_{\text {nint }}^{\prime}$ generated by the level density of back shifted Fermi gas model.

window to the canonical partition function is calculated in the FT-VAP approach and by direct diagonalization, the latter being used to test the accuracy of FT-VAP results. The contribution of the states outside the pairing window is taken into account through the grand canonical partition function of a non-interacting system, which is reduced to the canonical representation by the saddle point approximation. With this calculation scheme we have evaluated the specific heat in the isotopes ${ }^{161,162} \mathrm{Dy}$ and ${ }^{171,172} \mathrm{Yb}$. It is thus shown that the pairing correlations have a significant influence on the specific heat, especially for temperatures below $T=0.5 \mathrm{MeV}$. The comparison with the non-interacting systems shows clearly that the pairing correlations contributes to the S-shape behaviour of the specific heat, as noticed in the data extracted from experiment.

Compared to the specific heat extracted from experimental data, the calculations predicts much smaller values at larger temperatures. It is shown that the FT-VAP approach is in fact able to predict results close to the experiment if the contribution of the high energy excitations is calculated not with the single-particle states provided by the mean field models but with the Back Shifted Fermi Gas model, as actually done when the experimen- tal specific heat is evaluated. The necessity to replace the non interacting partition function at large excitation energy by the BSFGM one, clearly points out the important role of continuum part of the spectrum, neglected in these calculations. The calculated specific heat can be also influenced by the low-lying collective states which are not accounted for by the Hamiltonian (1). These two issues will be analysed in a future study.

\section{Acknowledgments}

This work was supported by the French-Romanian IN2P3-IFIN agreement and by the Romanian Ministry of Education and Research through the grant Idei nr 57.

\section{Appendix A: Canonical partition function for non-interacting particles}

We consider a system formed by a finite number of fermions distributed in a set of single-particle states of energy $\epsilon_{i}$, generated, for instance, by a self-consistent HF calculation. If there are no residual interaction between the particles, the partition function in the gran canonical ensemble can be written as

$$
\ln Z_{\text {nint }}^{G C}(\beta, \mu)=\sum_{i} \ln \left[1+e^{-\beta\left(\epsilon_{i}-\mu\right)}\right],
$$

where $\mu$ is the chemical potential.

The grand-canonical partition function can be used to obtain a simple approximation for the partition function in the canonical ensemble, expressed in terms of singleparticle quantities. This can be done by applying the saddle point approximation. One thus gets the following approximation for the canonical partition function [6]

$$
\ln Z^{\prime} \approx \ln Z^{\mathrm{GC}}+\beta E_{0}-\beta \mu N-\frac{1}{2} \ln \left(2 \pi\left\langle(\Delta N)^{2}\right\rangle\right)
$$

The chemical potential $\mu$, the particle number $N$ and its fluctuation $\Delta N$ are given by the equations

$$
N=\sum_{i} f_{i}, \text { and }\left\langle(\Delta N)^{2}\right\rangle=\sum_{i} f_{i}\left(1-f_{i}\right)
$$

where $f_{i}=\left[1+e^{\beta\left(\epsilon_{i}-\mu\right)}\right]$ is the Fermi-Dirac distribution. From the canonical partition function one can calculate the specific heat by doing numerical derivatives or using the formulas (B4, C1-C3) of Ref [6].
[1] E. Melby, et al., Phys. Rev. Lett. 83, 3150 (1999).

[2] D. Gambacurta, D. Lacroix, Phys. Rev. C85, 044321 (2012).

[3] A. Schiller, A. Bjerve, M. Guttormsen, M. Hjorth-Jensen, F. Ingebretsen, E. Melby, S. Messelt, J. Rekstad, S. Siem, S.W. Odegard, Phys. Rev. C 63, 021306 R (2001).

[4] A. Gilbert and A.G.W. Cameron, Can. J. Phys. 43, 1446
(1965).

[5] A. Bohr and B. Mottelson, Nuclear Structure, Vol. 1 (Benjamin, NY, 1969).

[6] Y. Alhassid, G.F. Bertsch, L. Fang, Phys. Rev C 68, 044322 (2003).

[7] V. Martinez, J.L. Egido and L.M. Robledo, Phys. Rev. C 68, 034327 (2003); E. Khan, N. Van Gian and N. Sand- 
ulescu, Nuclear Physics A 789, 94 (2007); A. F. Fantina, J. Margueron, P. Donati and P.M. Pizzochero, J. Phys. G: Nucl. and Part. Phys. 38, 025101 (2011); J. Margueron and E. Khan, Phys. Rev. C 86, 065801 (2012)

[8] T. Sumaryada and Alexander Volya Phys. Rev. C 76, 024319 (2007).

[9] N. Quang Hung and N. D. Dang, Phys. Rev. C 81, 057302 (2010); N. Quang Hung and N. D. Dang, Phys. Rev. C $82,044316(2010)$

[10] M. Bender, P.-H. Heenen, P.-G. Reinhard, Rev. Mod. Phys. 75, 121 (2003).

[11] C. Esebbag and J. L. Egido, Nucl. Phys. A 552, 205 (1993).

[12] P. Bonche, H. Flocard, and P.-H. Heenen, Comput. Phys. Commun. 171, 49 (2005).

[13] E. Chabanat, P. Bonche, P. Haensel, J. Meyer and R. Schaeffer, Nucl. Phys. A 635231 (1998).

[14] W. Long, J. Meng, N. Van Giai, and S.-G. Zhou Phys. Rev. C 69, 034319 (2004)
[15] Liu Lang, private communication

[16] M. Guttormsen, A. Bjerve, M. Hjorth-Jensen, E. Melby, J. Rekstad, A. Schiller, S. Siem, and A. Belic Phys. Rev. C 62, 024306 (2000).

[17] A. Schiller, et al, Phys. Rev. C 63, 021306 (2001).

[18] K. Esashika, H. Nakada and K. Tanabe, Phys. Rev. C 72, 044303 (2005)

[19] M. Guttormsen et al. Phys. Rev. C 62, 024306 (2000)

[20] K. Kaneko et al., PRC 74, 024325 (2006)

[21] A. L. Goodman, Nucl. Phys. A352, 30 (1981).

$[22]$ T. Duguet and G. Hagen, Phys. Rev. C 85, 034330 (2012).

[23] Usually the energy are measured with respect to the ground state energy $E_{0}$, it is often convenient to introduce the so-called excitation partition function $Z^{\prime} \equiv$ $Z e^{\beta E_{0}}$. [6] Then, $E_{n}$ correspond to the energy with respect to the ground state energy. 